\title{
Studies of Molecular Motions in Acylchitin Films by Means of Spin-Probe Technique
}

\author{
Katsuaki KAIFU,* Takashi KoMAI, ${ }^{* *}$ and Akihiro TSUTSUMI \\ Department of Polymer Science, Faculty of Science, \\ Hokkaido University, Nishi 8-chome, Kita 10-jo, \\ Kita-ku, Sapporo 060, Japan
}

(Received April 16, 1982)

\begin{abstract}
The molecular motions in acylated chitins, including acetyl, propionyl, butyryl, caproyl, capryl, lauroyl, and benzoylchitin, were investigated by ESR measurements with 2,2,6,6tetramethyl-4-hydroxypiperidine-1-oxyl (TEMPO) as a spin-probe. Introduction of a longer side chain into the chitin molecule resulted in a remarkable lowering of the temperature at which the separation between the outer two extrema of the ESR spectrum decreases adruptly $\left(T_{\mathrm{N}}\right)$. Below and above $T_{\mathrm{N}}$, two kinds of motional process were observed, one at a lower temperature having a smaller activation energy and the other at a higher temperature with a larger activation energy. These processes are a small scale motion such as rotational oscillation and a large scale motion such as rotation, respectively. The activation energy of the latter process decreased with increasing chain length, except for acetylchitin. Fully acetylated chitin films were anomalous with respect to the value of the activation energy.

KEY WORDS Acylchitin / Molecular Motion / Spin-Probe / ESR / Motional Process /
\end{abstract}

Chitin is a mucopolysaccharide consisting of a chain of 2-acetamide-2-deoxy-D-glucoses linked by $\beta-1,4$ bonds, and widely distributed in living organisms such as invertebrates, fungi, and molds. The $N$-acetyl-D-glucosamine units of chitin have two reactive hydroxy groups at the C-3 and C- 6 positions. Recently, a very good and easily applied procedure for the acetylation of chitin has been developed and reported by Nishi et al. ${ }^{1}$ Using this technique, we successfully prepared a series of acylchitin derivatives having aliphatic side chains of different lengths., ${ }^{2,3}$

Acylated chitin derivatives have particularly interesting physical and chemical properties. For example, acylchitins having longer aliphatic chains, such as butyryl, or caproyl, were readily soluble in ordinary organic solvents, whereas chitin, formylchitin, acetylchitin, and propionylchitin were soluble only in acidic solvents, such as formic acid or dichloroacetic acid.
Acylated chitins have been examined as materials for blood contact surfaces. ${ }^{4}$ Their critical surface tentions varied from 20 to $30 \mathrm{dyn}^{\mathrm{cm}}{ }^{-1}$, which suggests good blood compatibility. It is thus of considerable interest to investigate the biomedical significance of acylated chitins.

The spin-probe technique has been employed to obtain information about the degree of motional freedom in molecular systems through its application to ESR spectra of mixed paramagnetic probes. $^{5-8}$ In this article, we applied this technique to acylated chitin derivatives using a nitroxide radical as a probe suitable for investigating the dynamic properties of polymer solids. ${ }^{8}$ The results are correlated with the length of acyl side chains.

\section{EXPERIMENTAL}

\section{Materials}

2,2,6,6-Tetramethyl-4-hydroxypiperidine was

* Present address: Research Institute, Oki Electric Industry Company Ltd., 550-5 Higashisakawa-cho, Hachioji-shi Tokyo 192, Japan.

** To whom correspondence should be addressed. 
Table I. Structural formulas of acylchitins and that of the nitroxide radical

\begin{tabular}{ll} 
Chitin & $-\mathrm{H}$ \\
Formylchitin & $-\mathrm{COH}_{3}$ \\
Acetylchitin & $-\mathrm{COCH}_{3}$ \\
Propionylchitin & $-\mathrm{COCH}_{2} \mathrm{CH}_{3}$ \\
Butyrylchitin & $-\mathrm{COCH}_{2} \mathrm{CH}_{2} \mathrm{CH}_{3}$ \\
Caproylchitin & $-\mathrm{COCH}_{2} \mathrm{CH}_{2} \mathrm{CH}_{2} \mathrm{CH}_{2} \mathrm{CH}_{3}$ \\
Caprylchitin & $-\mathrm{COCH}_{2} \mathrm{CH}_{2} \mathrm{CH}_{2} \mathrm{CH}_{2} \mathrm{CH}_{2} \mathrm{CH}_{2} \mathrm{CH}_{2} \mathrm{CH}_{2} \mathrm{CH}_{3}$ \\
Lauroylchitin & $-\mathrm{COCH}_{2} \mathrm{CH}_{2} \mathrm{CH}_{2} \mathrm{CH}_{2} \mathrm{CH}_{2} \mathrm{CH}_{2} \mathrm{CH}_{2} \mathrm{CH}_{2} \mathrm{CH}_{2} \mathrm{CH}_{2} \mathrm{CH}_{3}$ \\
Benzoylchitin & $-\mathrm{COC}_{6} \mathrm{H}_{5}$ \\
\hline
\end{tabular}

$2,2,6,6,-$ Tetramethy $1-4-$ hydroxy-
piperidine-1-oxy 1

(TEMPO)<smiles>CC1(C)CC(O)CC(C)(C)N1O</smiles>

purchased from Aldrich Chemical Co. Formic acid and chloroform for casting films were purchased from Wako Pure Chemical Industries Ltd., and purified by distillation.

\section{Sample Preparations}

The spin-probe used in this work was 2,2,6,6tetramethyl-4-hydroxypiperidine-1-oxyl (TEMPO). It was prepared by oxydizing 2,2,6,6-tetramethyl-4hydroxypiperidine with hydrogen peroxide in the presence of sodium tungstate and EDTA, using a procedure similar to that reported by Rozantzev et al. ${ }^{9}$

Films of chitin, acetylchitin, and propionylchitin were obtained by casting their formic acid solution onto glass plates. The films were thoroughly washed with distilled water and dried. They were immersed in a $1 \%$ aqueous solution of TEMPO for one week and then washed with water, dried in vacuo for three days at $50^{\circ} \mathrm{C}$. Films of butyrylchitin, caproylchitin, caprylchitin, lauroylchitin, and benzoylchitin were prepared by casting their chloroform solutions each containing $10^{-3}$ equiv. mole of TEMPO per glucosamine residue of chitin derivatives. These films were dried in vacuo for three days at $50^{\circ} \mathrm{C}$. One of the spin-probed caprylchitins was also prepared by the penetrative procedure as in the case of chitin, acetylchitin and propionylchitin.

In Table I, the structural formulas of acylchitins and the nitroxide radical are illustrated. The degree of acyl substitution per $N$-acetylglucosamine residue of the chitin was 1.4 for formylchitin, 2.0 for acetylchitin, 1.9 for propionylchitin, 1.8 for butyrylchitin, 2.0 for caproylchitin, 1.8 for caprylchitin, 1.9 for lauroylchitin, and 1.9 for benzoylchitin.

\section{ESR Measurement}

The spin-probed acylchitin films were packed in $5 \mathrm{~mm}$ quartz sample tubes and sealed in vacuo. ESR spectra were obtained by a JEOL ESR spectrometer, Model JES-FE1X over a temperature range from -150 to $170^{\circ} \mathrm{C}$. 


\section{RESULTS AND DISCUSSION}

The typical ESR spectra for caproylchitin at $-140,-15,32,80$, and $158^{\circ} \mathrm{C}$ are shown in Figure 1. At lower temperatures, the spectra consist of a broad and asymmetric triplet, indicating low mobility of the probe. As the temperature is raised, the spectra become narrow and, near room temperature, a second narrow component appears superimposed on the broad component. At higher temperatures, the spectra consist only of a narrow component and become a sharp triplet, indicating very fast motion of the probe.

Separation of the outer extrema $(W)$ is plotted against temperature in Figure 2. For each sample, an abrupt narrowing occurs near room temperature. However, the narrowing regions are different for different aliphatic side chains.

For convenience, the temperature at which $W$ of broad component is 60 gausses is taken as the narrowing temperature $\left(T_{\mathrm{N}}\right)$. Figure 3 shows the relationship between $T_{\mathrm{N}}$ and the length of the acyl groups in chitin derivatives. The length of an acyl

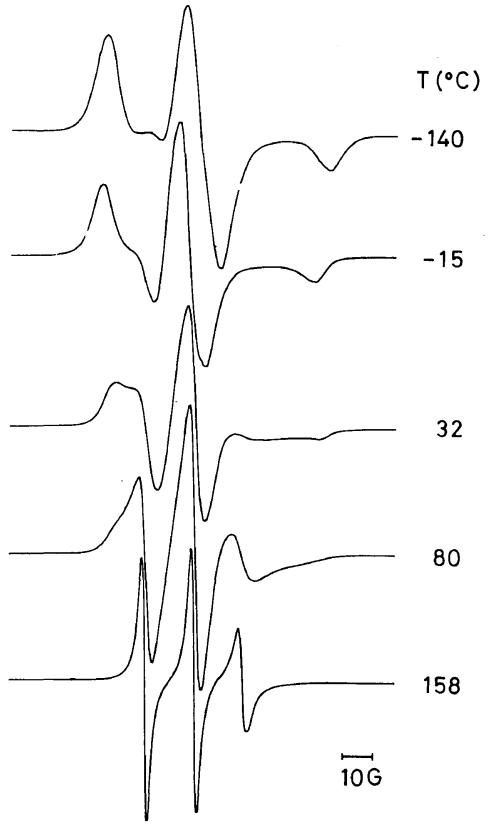

Figure 1. ESR spectra of spin-probed caproylchitin film at various temperatures.

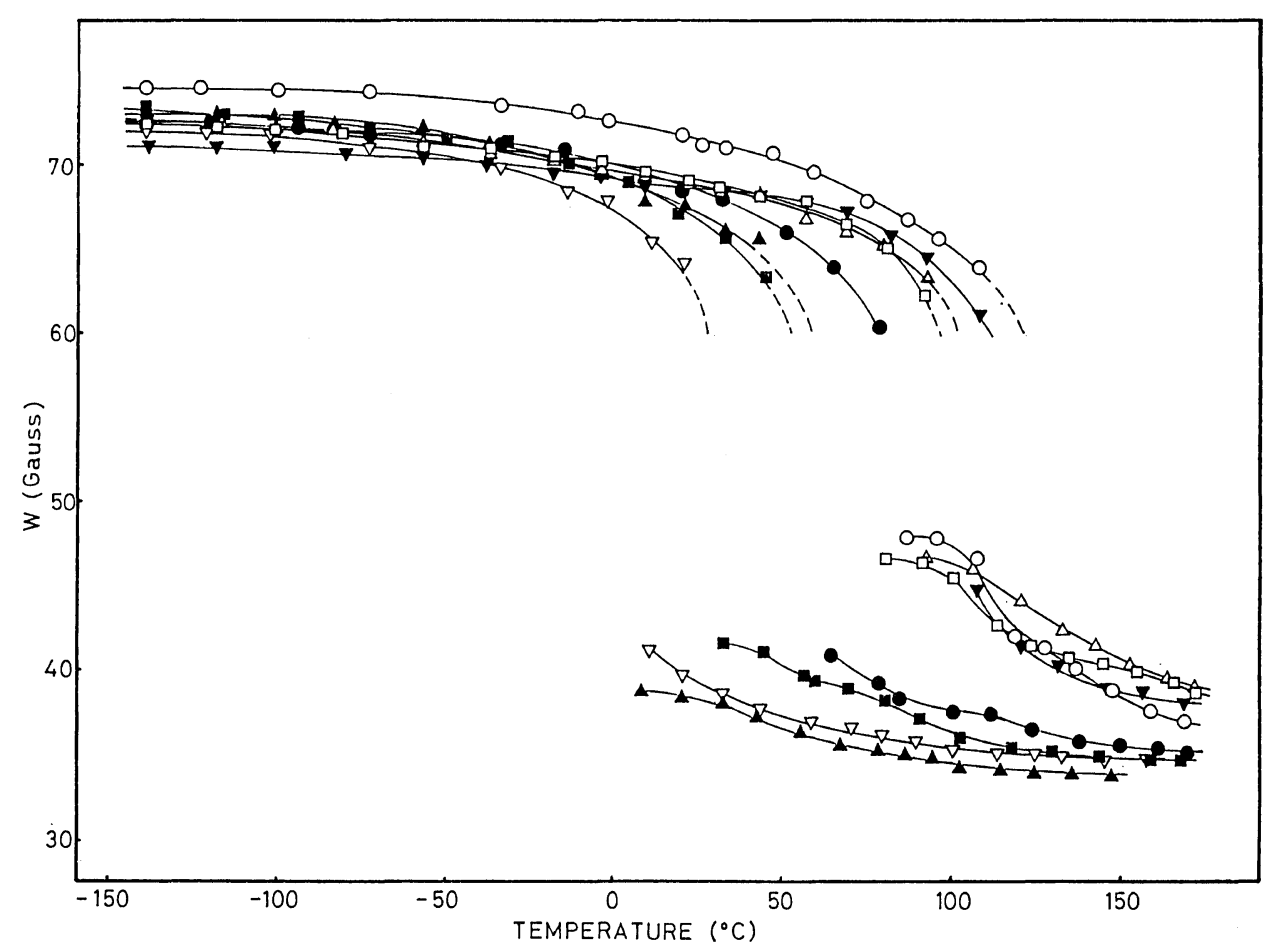

Figure 2. Temperature dependence of the extrema separation for chitin and acylchitins: $(\bigcirc)$, chitin; $(\square)$, 2.0 acetylchitin; $(\triangle), 1.9$ propionylchitin; $(\bigcirc), 1.8$ butyrylchitin; $(\boldsymbol{\Delta}), 2.0$ caproylchitin; $(\mathbf{\Delta}), 1.8$ caprylchitin; $(\nabla), 1.9$ lauroylchitin; $(\nabla), 1.9$ benzoylchitin. 
group is represented by the number of carbons, it contains: 0 for chitin, 2 for acetylchitin, and so on. The value of $T_{\mathrm{N}}$ decreases as the length of the acyl group increases.

The correlation time $\left(\tau_{c}\right)$ for the rotational motion of TEMPO was estimated as follows; At a higher temperature where the spectrum was suf-

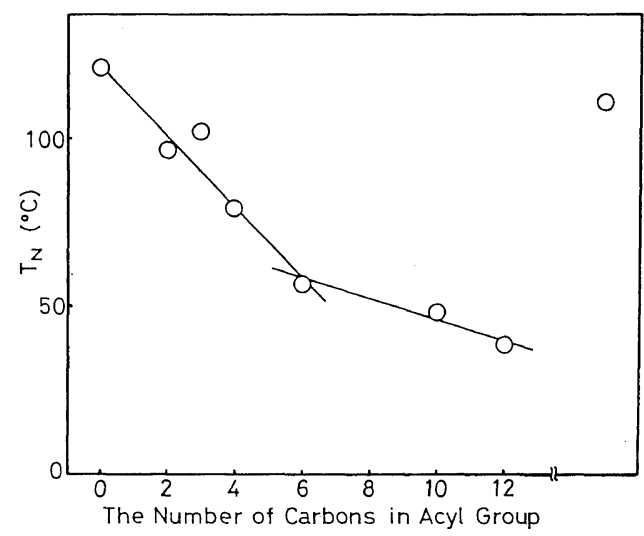

Figure 3. The relationship between the side chain length of acylchitins and $T_{\mathrm{N}}$.

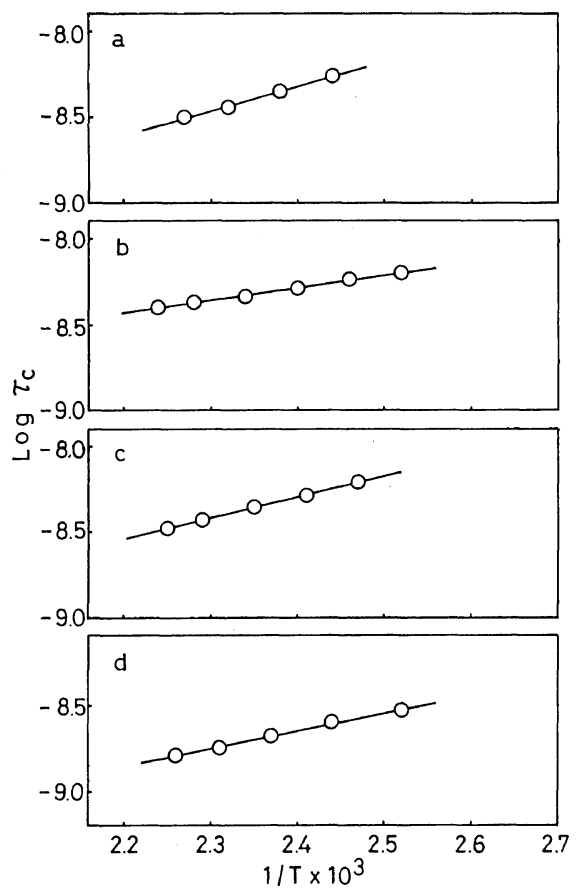

ficiently narrow, $\tau_{\mathrm{c}}$ was calculated using the equation based on the theory by Kivelson ${ }^{10}$ :

$$
\tau_{\mathrm{c}}=5.06 \times 10^{-10}\left[\left(h_{0} / h_{1}\right)^{1 / 2}+\left(h_{0} / h_{-1}\right)^{1 / 2}-2\right] W_{0}
$$

where $h_{1}, h_{0}$, and $h_{-1}$ are the heights of three peaks found in order from low magnetic field; $W_{0}$ is the width of the central peak in guasses. The numerical constant in eq 1 was calculated from the anisotropy of hyperfine constants for TEMPO. The Arrhenius plots of $\tau_{\mathrm{c}}$ are shown in Figure 4.

In the slow motional region corresponding $10^{-9}<\tau_{\mathrm{c}}<10^{-6} \mathrm{~s}$, the correlation time was calculated using the method developed by Freed et al. ${ }^{11}$ :

$$
\begin{aligned}
& \tau_{\mathrm{c}}=a \cdot(1-S)^{b} \\
& S=A_{\mathrm{z}}{ }^{\prime} / A_{\mathrm{z}}
\end{aligned}
$$

where $A_{z}{ }^{\prime}$ is the separation of outer extrema and $A_{z}$ is the rigid limit value for the same quantity. For the Brownian diffusion model, the values of parameters $a$ and $b$ have been estimated to be $5.4 \times 10^{-10} \mathrm{~s}$ and -1.36 , respectively. ${ }^{11}$

Figure 5 shows the Arrhenius plots of $\tau_{c}$ for acetylchitin in the slow motional region. The
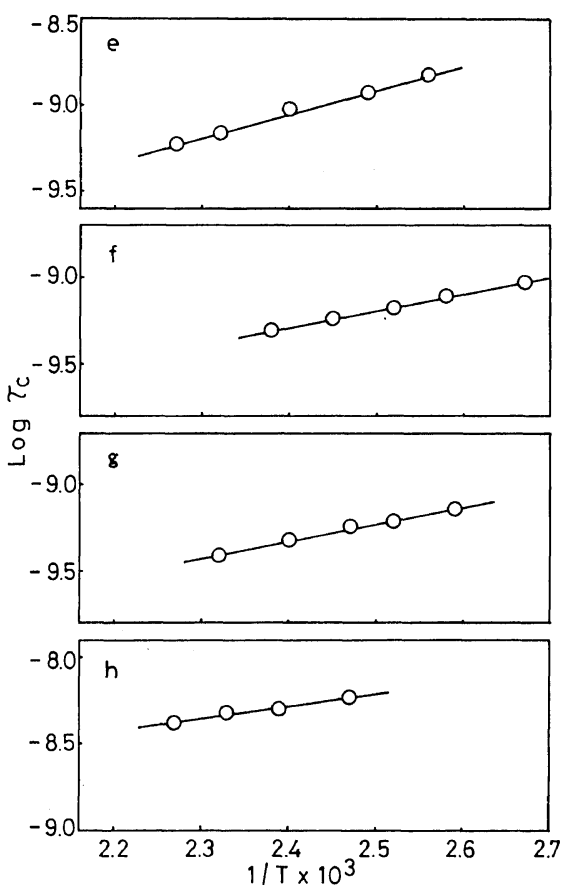

Figure 4. Arrhenius plots of the correlation time for chitin and acylchitins in the high temperature region: (a) chitin; (b) 2.0 acetylchitin; (c) 1.9 propionylchitin; (d) 1.8 butyrylchitin; (e) 2.0 caproylchitin (f) 1.8 caprylchitin; (g) 1.9 lauroylchitin; (h) 1.9 benzoylchitin. 
Arrhenius plots for other acylchitin derivatives were also linear.

The value of $\tau_{c}$ at $0^{\circ} \mathrm{C}$ and $135^{\circ} \mathrm{C}$ are plotted

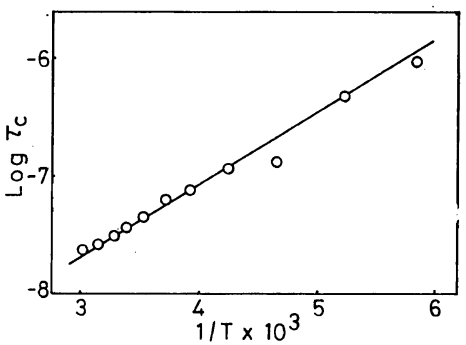

Figure 5. Arrhenius plots of the correlation time for 2.0 acetylchitin in the low temperature region.

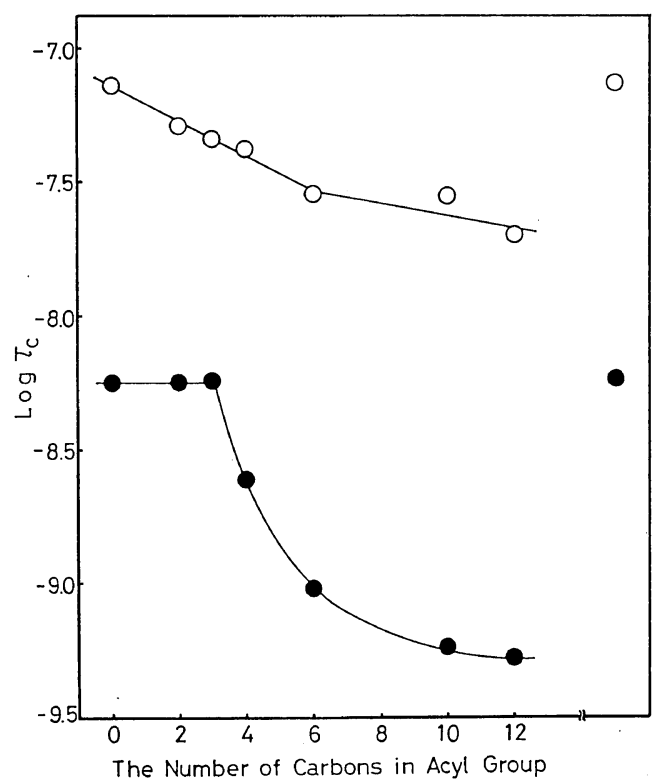

Figure 6. Relationship between the side chain length of acylchitins and $\log \tau_{\mathrm{c}}$ at $0^{\circ} \mathrm{C}(\mathrm{O})$ and $135^{\circ} \mathrm{C}(\mathrm{O})$.

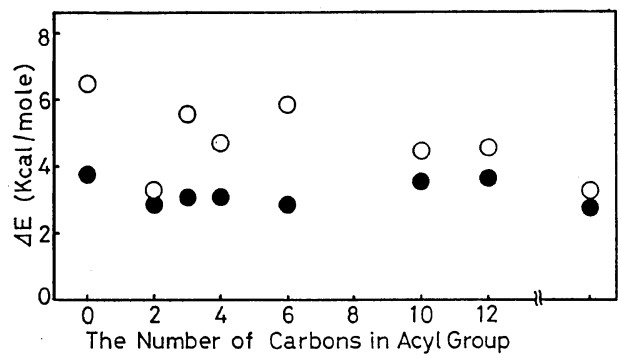

Figure 7. Relationship between the side chain length of acylchitins and activation energy at higher $(O)$ and lower (O) temperatures. against the length of the acyl groups in Figure 6.

At $0^{\circ} \mathrm{C}$, the value of $\tau_{\mathrm{c}}$ decreases monotonically with increasing side chain length. But at $135^{\circ} \mathrm{C}$, the $\tau_{\mathrm{c}}$ values are nearly equal for side chains with carbon number up to 3 , and then decrease for side chains whose carbon number is 4 .

The activation energy for the rotational motion of the spin-probe in various acylchitins was estimated from the straight lines in fast (Figure 4) and slow (Figure 5) motion regions, and is summarized in Figure 7.

The activation energies in the low temperature

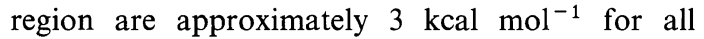
samples, and those in the higher temperature region

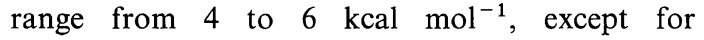
acetylchitin.

The spin-probed caprylchitin films were prepared by two different method: casting a chloroform solution of TEMPO and immersion of the film in an aqueous solution of TEMPO. No differences in temperature dependence of the extrema separation and the correlation time were found in the caprylchitin films thus prepared. Therefore, the results obtained above were not influenced by the differences in the method of sample preparation.

All the results indicate that molecular motions observed by the spin-probe method are considerably dependent on side chain length, and that the spin-probe monitors the motion in the side chain region of the acylchitins, as in the case of $\alpha$-helical poly(amino acids). ${ }^{12}$ The side chain region becomes more mobile with an increase in side chain length, except for benzoylchitin, in which the side chain motion is probably restricted owing to the bulkiness of the phenyl group and is not much different from that of original chitin.

It has been reported that the molecular motion of the spin-probe involves different processes such as oscillation, rotation, and translation. ${ }^{8}$ Our data for activation energy indicate that there are two types of processes for TEMPO in acylchitins, one at lower temperatures having a smaller activation energy and another at higher temperatures having a larger activation energy. These processes correspond to a small scale motion such as oscillation and a large scale one such as rotation or translation. Figure 6 suggests that the amplitude of the small scale motion increases with an increase in the acyl side chain length.

It has been shown that at temperatures above $T_{\mathrm{N}}$, 
the activation energies of a spin-probe in ordinal synthetic polymers sometimes are higher than 10 $\mathrm{kcal} \mathrm{mol}{ }^{-1}$ owing to the translational diffusion process throughout the polymer matrix. ${ }^{13}$ In acylchitin, the activation energies were much smaller than $10 \mathrm{kcal} \mathrm{mol}^{-1}$, suggesting that the process is rotational. It may be concluded that the degree of motional freedom in the side chain region of chitin derivatives is not high enough to cause translation or jump of the spin-probe because of the relatively rigid structure of the main chain.

An interesting fact can be seen for acetylchitin with a carbon number of 2 . This sample has the smallest activation energy among acylchitins in the higher temperature region. Corresponding to this, an anomaly was found in the wetting characteristics and blood clotting time of acetylchitin. ${ }^{4}$ The dispersive and non-dispersive components of the surface tension of chitin derivatives with 2.0 acetyl chitin were very close to those obtained for glutaraldehyde treated umbilical cord vessels widely used as artificial blood vessels. ${ }^{14}$ The clotting time on the surface of these derivatives was twice that on the siliconized glass. However, clotting time on all other acylated chitin suface was less than on the siliconized glass, though more than on the original chitin surface.

At present, no adequate explanation of these anomalies is available. The acetylchitin contains no free hydroxy groups, and the hydrophobic interaction among molecule would therefore be smaller than other acylchitins having longer aliphatic side chains. This fact may lead to the formation of a specific structure on a solid surface. Anomalies found in blood clotting time, wetting characteristics, and activation energy of a spin-probe in a solid phase may be attributed to this specific surface structure.

Acknowledgements. This work was supported in part by a Grant-in-Aid for the Scientific Research (Medical Polymer) from the Ministry of Education,
Science and Culture of Japan, for which the authors express their sincere gratitude.

The authors are also indebted to Dr. N. Nishi at the Department of Polymer Science, Faculty of Science, Hokkaido University for his valuable comments and assistance in the preparation of acylchitins.

\section{REFERENCES}

1. N. Nishi, J. Noguchi, S. Tokura, and H. Shiota, Polym. J., 11, 27 (1979).

2. K. Kaifu, N. Nishi, T. Komai, S. Tokura, and O. Somorin, Polym. J., 13, 241 (1981).

3. K. Kaifu, N. Nishi, and T. Komai, J. Polym. Sci., Polym. Chem. Ed., 19, 2361 (1981).

4. K. Kaifu and T. Komai, J. Biomed. Mater. Res., 16, in press, T. Komai, K. Kaifu, N. Nishi, J. Noguchi, and M. Nakazono, Preprints, the 8th Symposium on Biomedical Polymers, Kyoto, 1978, Japan, p 21; K. Kaifu and T. Komai, Preprints, the 9th Symposium on Biomedical Polymers, Osaka, 1979, Japan, p 13; T. Komai, K. Kaifu, T. Kawasaki, M. Matsushita, T. Kawamata, M. Kondo, A. Kawamura, T. Kon, I. Koshino, and Y. Kasai, Preprints, the 10th Symposium on Biomedical Polymers, Tokyo, 1980, Japan, p 39.

5. V. B. Stryukov and E. G. Rozntzev, Vysokomol. Soedin. A., 10, 626 (1968).

6. G. P. Rabold, J. Polym. Sci. A-1, 7, 1187 (1969).

7. G. P. Rabold, J. Polym. Sci. A-1, 7, 1203 (1969).

8. N. Kusumoto, "Molecular Motion in Polymers by ESR," R. F. Boyer and S. E. Keinath, ed, Harwood Academic Publishers, New York, 1980, p 233.

9. E. G. Rozantzev and L. A. Krinitzkaya, Tetrahdedron, 21, 491 (1965).

10. D. Kivelson, J. Chem. Phys., 33, 1094 (1960).

11. J. H. Freed, V. Bruno, and C. Polnaszek, J. Phys. Chem., 75, 3385 (1971).

12. A. Tsutsumi, K. Hikichi, and M. Kaneko, Polym. J., 8, 511 (1976).

13. S. A. Goldman, G. V. Bruno, and J. H. Freed, J. Phys. Chem., 76, 1858 (1976).

14. T. Komai, Y. Sawada, Y. Onda, I. Koshino, Y. Nakanishi, A. Nishimura, and Y. Kasai, Artif. Org. (Official J. Internat. Soc. Artif. Org.), 2 (Suppl), 115 (1978). 